\title{
A Situated Game for Autistic Children Learning Activities of Daily Living
}

\author{
Maiga Chang \\ School of Computing and Information Systems \\ Athabasca University \\ Canada \\ maiga@ms2.hinet.net
}

\author{
Chun-Wei Lyu, Jia-Sheng Heh \\ Department of Information and Computer Engineering \\ Chung-Yuan Christian University \\ Taiwan \\ kobe0520@yahoo.com.tw,jsheh@ice.cycu.edu.tw
}

\author{
Rita Kuo \\ Academic and Industrial Research Centre \\ Knowledge Square, Inc. \\ Taiwan \\ rita.mcsl@gmail.com
}

\begin{abstract}
Daily living skills are difficult for autistic children to learn because they have low motivation in learning new things. Some research has developed virtual environment to assist parents and teachers teaching autistic children daily living skills, educators still need to spend a lot of time in preparing personalized and more realistic tasks for children to practice. This research designs an activity generation mechanism by measuring activity's weight with fuzzy theory and rough set's help. Based on the activity generation mechanism and weight measurement, a Flash-based situated game is developed for providing autistic children personalized and non-repeated practices of activities of daily living. An evaluation plan of the pilot for verifying the effectiveness of the game and gathering the users' (include teachers, parents, and the autistic children) perceptions toward the game and the game-play is designed.
\end{abstract}

Keywords- Game-based learning, autism, children, preadolescence, activities of daily living, knowledge structure, situated learning

\section{INTRODUCTION}

To live independently, we need to learn how to do some activities by ourselves, such as dressing and bathing. These activities usually have more than one step to do. Take dressing for example, we have to get clothes from closets and drawers first, then we put on clothes, outer garments, and manage fasteners [2]. These activities are called activities of daily living [3]. Activities of daily living are usually developed in our childhood [8]. However, parents having autistic children report that they have difficulty in teaching their children to do activities of daily living [4].

To help autistic children learn the procedure of doing activities of daily living, Pierce and Schreibman (1994) use photo books for displaying the procedures of activities of daily living [7]. The educators take pictures for each step of activities and each photo book can be used to teach children the procedure of one activity of daily living. Autistic children can follow the pictures in the photo books and do the activities of daily living in the authentic environment. Shipley-Benamou and colleagues (2002) take videos of the procedures of doing activities, such as making orange juice and mailing a letter, and ask autistic children to learn the procedures by watching the videos before they do the activities in the authentic environment [9]. The experiment results show that autistic children can learn the procedures effectively through watching videos and remember the procedures after a month without watching the videos again.

Autistic children may make mistakes when they do activities of daily living in the authentic environment. Some mistakes may hurt autistic children themselves, such as cutting their fingers by the broken cup. To make the authentic environment safer to autistic children, Charitos and colleagues (2000) use virtual reality technology to build a virtual house with five rooms, including bathroom, kitchen, child's room, parents' room, and living room [1]. The scenario starts when the avatar comes home. Autistic children can control the avatar to do different activities in the proper room, for instance, washing hands in the bathroom, setting table in the kitchen, dressing themselves in parents' room, helping parents clean the living room, and making his/her bed in the child's room [6]. If autistic children don't know the correct procedures of doing activities, they can choose animation mode to watch animations played by the virtual character in order to learn how to do the activities [1].

However, children may have problem in adopting the procedure that they had learnt in real life, because the real world may be different from the authentic environment or virtual house. For example, the activities of "having breakfast" and "having lunch" are similar, and their procedures are "taking the tableware to the table from the cupboard", "putting foods onto the plate", "feeding yourself", "taking the tableware to the kitchen sink", "washing the tableware", and "putting the tableware back to the cupboard". Once John, an autistic child, forget to do one step in the "having breakfast" activity, such as forgetting "cleaning the dish (one of the tableware used in the activity)", the procedures of doing the next activities in the real world are needed to be changed a little bit.

In the real world, the unwashed dish is needed for "having lunch" activity, and it is needed to be washed before John putting foods on it. In other words, the procedure of "having lunch" activity is then influenced by the dish's status. 
John needs to insert "cleaning the dish" in the beginning of the original procedure of "having lunch" in order to fit the new situation. The pedagogical methods used in the previous research can not change the instructional materials to adopt the new situation automatically. Educators need to put new photos in photo books, take another video, or create new animation for the new situation. All these works take time and increase educators' burden.

To solve problems mentioned above, the objective of this research is to design a web-based Flash educational game which can generate activities automatically to give children immersive learning experience based on the time in the game world as well as the object's status, for example, it would be weird to ask children to do "having breakfast" activity in the morning of a day in the game; or to ask children to "clean dinning table" when there is nothing on the table. To reach the goal, we need to consider the solutions of two issues:

- Issue \#1: how to store the activities of daily living and relevant objects in the real world?

- Issue \#2: how to generate activity of daily living for children to give them personalized immersive and nonrepeated learning experience in the game world?

To solve the two issues, this research first analyzes the attributes of activities of daily living and uses final state automata to store and represent the relations among activities, and rough set to classify and represent activity relevant objects. Secondly, with the help of fuzzy theory and selfdesigned weighting measures, the research is capable of picking up most appropriate activity for children according to the time in the game and all objects in the virtual world. This mechanism not only makes children practice what they have leant, but also gives them opportunities to see more real situations which are either caused by incomplete procedure they had done previously or being realistic scenario as real world has; their ability of doing activities of daily living can then be sharpened further by having such chance to apply every possible skill that they have leant to solve real world complex situation.

This paper is organized as follows. Section 2 introduces the finite automata used to store and represent the relations among activities. The fuzzy-based activity generation mechanism and the weighting measures are designed and discussed in Section 3. Section 4 shows the game and Section 5 discusses the evaluation plan that we plan to verify the usability of this game.

\section{ACTIVITIES IN THE GAME WORLD}

The relations of activities of daily living can be presented by a finite state automaton as Fig. 1 shows. During weekdays, we "have lunch" after "breakfast". If the day is Wednesday, the next activity of "having lunch" is "cleaning living room". After reach the state of "going to sleep", the machine will go back to the initial state of the automaton, i.e., "eating breakfast".

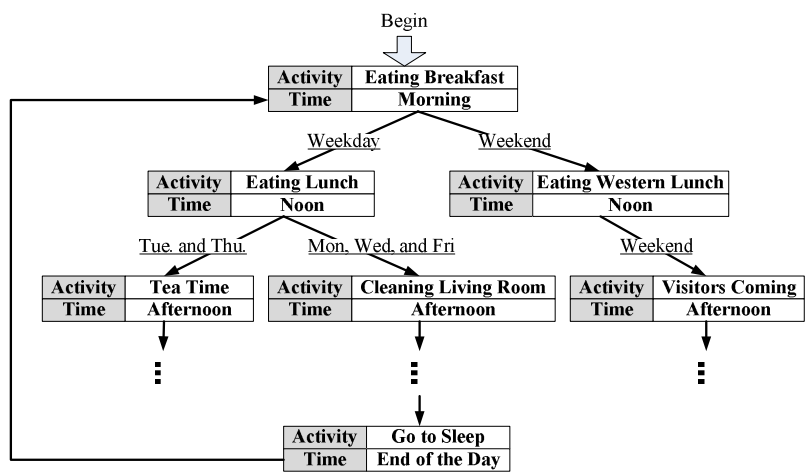

Figure 1. Finite state automaton presents the activities of daily living.

To accomplish an activity of daily living, children must do a series of tasks, i.e., the procedure of doing activity of daily living. For example, children have to "wash hand" first according to the procedure of "eating breakfast" activity. After that, they have to "prepare the tableware" before having breakfast on the table and "clean the table" after they finish their breakfast. Once the children complete all tasks in the procedure, the activity is considered complete and they are ready to do another activity.

Each task in the procedure will change the object status in the game world as it always does in the real world. Each object in the procedure has its required final-status for completing the activity. For example, the fork has to be clean and placed on the table for "eating breakfast". And yes, an activity may cover more than one stage such like "prepare tableware", "clean the table", and "wash the dishes" in the "eating breakfast" activity.

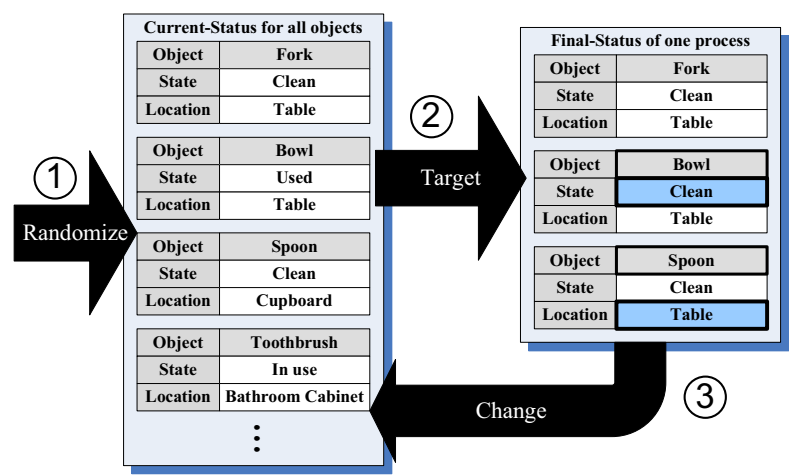

Figure 2. Example of random-status of all objects and final-status requirement of "prepare tableware" stage.

Fig. 2 shows an example of the concept of object's status changes when the children do an activity. First of all, the game generates objects' status including their state and location randomly. In this example, as we can tell from the final-status requirement on the right of Fig. 2, the goal of this stage is to wash the fork, bowl and spoon and then place them on the table.

However, the bowl is used and on the table right now and the spoon is still in the cupboard. In this case, the children need to clean the bowl and put the bowl back on the table, and, furthermore, to bring the spoon from the cupboard to the 
table. Once the children completed both tasks, the currentstatus of fork, bowl, and spoon will meet the required finalstatus of this stage. The new current-status can be used for the next process to generate new quests.

To make children receive personalized and non-repeated real life like activities, this research first randomizes the status of all objects in the game world at the very beginning when children start to play. By playing the game, the children will receive different quest that asking them to do particular activity according to the time in the game world as well as the object status in the virtual world. The children's actions in doing different activities may make their game worlds become more differently from others, those different game worlds lead them to receive different activities even further.

\section{ACTIVITY GENERATION}

With the help of finite state automaton and required object's final status settings, we can store the activities of daily living and provide children quests of doing variety of activities in the game world. However, as we can tell from Fig. 1, actually we have two further questions come out, they are which activity should be chose to ask the children doing and what should be the follow-up activity once the children finish one.

This research uses time in the game world as a trigger and uses fuzzy membership function value to weight activities. Fig. 3 shows partial of activities of daily living, in this figure, we can see two activities: "eat breakfast" and "eat lunch". Although from seven to nine is the time period of "eating breakfast", sometimes people may have their breakfast a little bit of earlier or later. So the fuzzy membership values in-between nine to eleven and earlier than seven are still larger than zero and less than one.

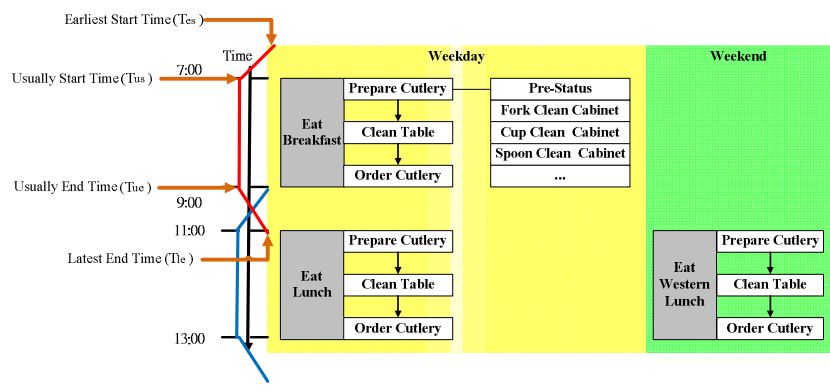

Figure 3. Time-based activities of daily living and its fuzzy membership functions.

Beside the time-based fuzzy membership values which we can calculate for every possible activities according the current time in the game world, we still need to consider the object's status as well for generating activities for children. The reason that we can not just count on the time and its fuzzy membership value is because of "learning". The game's goal is to help autistic children learn how to do activities of daily living, so it would be meaningless if the game picks up an activity that the children don't need to do anything further, for instance, the children don't need to "prepare the tableware" for "eating breakfast" if the table in the game world already has clean fork, bowl, and spoon on itself.

This research tries to categorize all objects and its status into rough sets - positive set, boundary set, and negative set, based on activities as Fig. 4 shows.

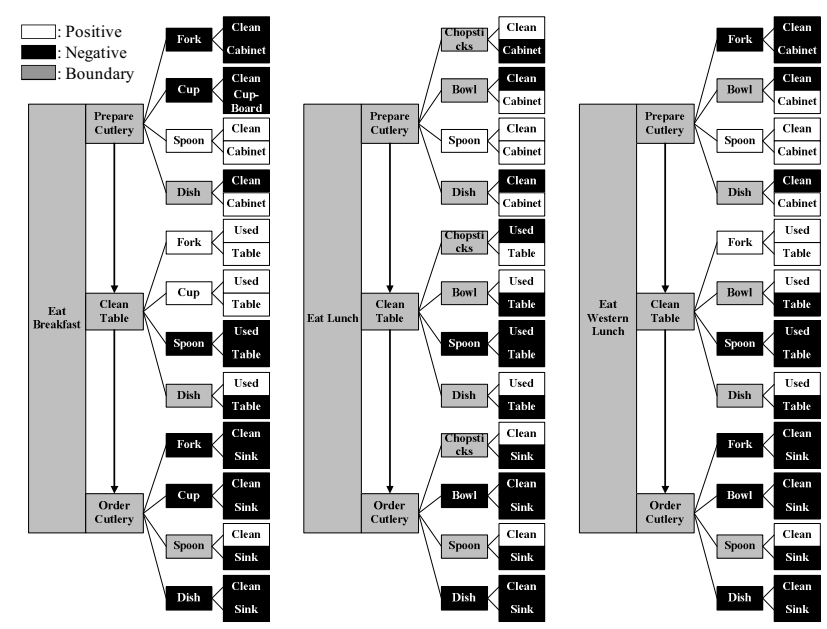

Figure 4. Using rough sets to categorize objects based on activities of daily living and its stages.

In such case, we can count how many negative and boundary set items that each activity (and its stage) has, in order to know how complex the tasks would be to complete the stage (or the whole activity) when the children are being asked to do the particular activity.

By combing both of the time-based fuzzy membership values (i.e., time-based weight) and the object status based rough set ratio (i.e., object status based weight), the game can pick-up appropriate activity for the children and the activity is personalized and non-repeated one.

\section{THE GAME}

With the proposed activity generation mechanism, we develop a Flash-based situated game which generates personalized quests for the children (i.e., particularly the autistic children in this research) automatically. When children enter the game in the beginning, the game will randomly generate status for all objects as well as the time in the game world. The game then calculates the time-based weights for all activities and picks activity candidates by ignoring those activities whose time-based weight value is zero. For those activity candidates, the game further measures its rough set ratios according to the current status that associated objects have. At the end, the game chooses one activity from the candidates and asks the children to complete the correspondent tasks as Fig. 5 shows.

The game is developed by Flash ActionScript 3.0 and connects to mySQL database via Java Server Pages. The mySQL database stores eleven activities in four rooms: dinning room (with kitchen), living room, bedroom, and washroom. Fig. 5 shows an activity in the dining room. 


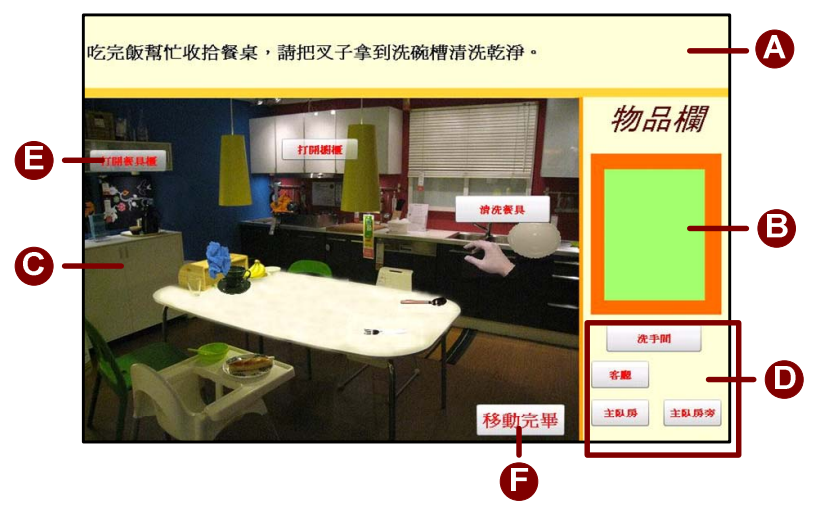

Figure 5. An activity in the dinning room.

The activity in Fig. 5 is "You have finished breakfast, please help mother clean the table and wash the fork in the sink." The activity description is shown in area A, which asks the children to bring the fork to the sink in the kitchen and wash it. The dinning room is shown in area $\mathrm{C}$, in which some moveable objects like spoon and fork are there. If users want to go to the other room, they can click the buttons with room name in area $\mathrm{D}$ or the adjacent room button (i.e., button E) in the main scene. The children can move objects to any place of this room directly. If they want to move objects to another room, then they need to move the objects to area B first before they go to anther room. When the children complete the activity, they can click button $\mathrm{F}$ to tell the game in order to start next one.

\section{Evaluation Plan}

The research has done experiment design to verify if the game is useful to autistic children. To make autistic children fill comfortable during the experiment, the experiment location is their home. The entire experiment plans to spend four weeks and the tasks in every week are listed in Table I.

TABLE I. TASKS IN EVERY WEEK

\begin{tabular}{|c|c|}
\hline Week & Tasks \\
\hline 1 & $\begin{array}{l}\text { - Parents sign consent letter } \\
\text { - Children do pre-test } \\
\text { - Parents help children fill questionnaire } \\
\text { - Introduce the game to parents and children } \\
\text { - 1st game play }\end{array}$ \\
\hline 2 & - 2nd game play \\
\hline 3 & - 3rd game play \\
\hline 4 & $\begin{array}{l}\text { - } 4 \text { th game play } \\
\text { - Children do post-test } \\
\text { - Parents fill questionnaire } \\
\text { - Parents help children fill questionnaire } \\
\text { - Interview with parents }\end{array}$ \\
\hline
\end{tabular}

The consent form is going to ask parents to sign in the first week before we start the experiment. In the consent form, we also explain the experiment purpose, spending time, and the required gaming environment. Children are going to do 15 multiple-choice items related to the activities of daily-living as the pre-test. Furthermore, a questionnaire has five parts (include demographics, computer game attitude, digital literacy, computer anxiety, and computer coping strategies) is asked children to fill up. Due to autistic children sometime may require help in filling questionnaire, we ask parents to be with them all the time during the experiment.

After the children filled questionnaire, we will give the children and their parents a user guide of the game play. Beside the user guide, we are also going to show them how to play the game and use the functions. In the first game play, autistic children are accompanied with parents and researchers. In the followed game plays, parents and researchers are asked to now help the children but only watch their game-play.

After the last game play, the children need to take posttest in which all items come from the pre-test but with different order. Also, we are going to ask both parents and children to fill up a questionnaire regarding their acceptance of such game. We will have interviews further with parents to get more detailed perceptions and suggestions toward the game and the usability of improving children's ability of doing activity of daily living.

\section{REFERENCES}

[1] Charitos, D., Karadanos, G., Sereti, E., Triantafillou, S., Koukouvinou, S., and martakos, D. (2000). Employing virtual reality for aiding the organisation of autistic children behaviour in everyday tasks. In the Proceedings of the 3rd International Conference on Disability Virtual Reality and Associated Technologies (pp. 147-152), September 23-25, 2000, Alghero, Italy.

[2] Iowa Geriatric Education Center (n.d.) Index of Independence in Activities of Daily Living (Katz Index of ADL). Retrieved October, 10, 2011, from The University of Iowa, Iowa Geriatric Education Center Website:

http://www.healthcare.uiowa.edu/igec/tools/function/katzADLs.pdf

[3] Katz, S., Ford, A.B., Moskowitz, R.W., Jackson, B.A. \& Jaffe, M.W. (1963). Studies of illness in the aged. The index of ADL: A standardised measure of biological and psychosocial function. Journal of the American Medical Association, 185(12), 914-919.

[4] Koegel, R. L., \& Egel, A. L. (1979). Motivating autistic children. Journal of Abnormal Psychology, 88, 418-426.

[5] Konstantinidis, E., Luneski, A., Frantzidis, C., Nikolaidou, M., Hitoglou-Antoniadou, M., Bamidis, P. (2009). Information and Communication Technologies for Enhanced Education of Children with Autism Spectrum Disorders. The Journal on Information Technology in Healthcare, 7(5), 284-292.

[6] Matson, J. L., Dempsey, T., and Fodstad, J. C. (2009). The effect of Autism Spectrum Disorders on adaptive independent living skills in adults with severe intellectual disability. Research in Develoopmental Disabilities, 30, 1203-1211.

[7] Pierce, K., \& Schreibman, L. (1994). Teaching daily living skills to children with autism in unsupervised settings through pictorial selfmanagement. Journal of Applied Behavior Analysis, 27, 471-481.

[8] Pinto, K. S. and Denucci, S. M. (2006) Activities of Daily Life. L. W. Braga, A. C. da Paz Jr. (Ed.), The child with traumatic brain injury or cerebral palsy: a context-sensitive family-based approach to development (pp. 173-200). London: Taylor \& Francis.

[9] Shipley-Benamou, R., Lutzker, J. R., \& Taubman, M. (2002). Teaching daily living skills to children with autism through instructional video modeling. Journal of Positive Behavior Interventions, 4, 165-175. 